\title{
Direct current electrical stimulation chamber for treating cells in vitro
}

\author{
Sahba Mobini', Liudmila Leppik, and John H. Barker \\ Frankfurt Initiative for Regenerative Medicine, Johann Wolfgang \\ Goethe-University, Frankfurt am Main, Germany
}

tPresent address: School of Materials, Faculty of Engineering and Physical Sciences, University of Manchester, Manchester, UK

BioTechniques 60:95-98 (February 2016) doi 10.2144/000114382

Keywords: DC electrical stimulation; in vitro; tissue engineering; bioreactors; osteogenic differentiation

Electrical stimulation has been shown to promote healing and regeneration in skin, bone, muscle, and nerve tissues in clinical studies. Recently, studies applying electrical stimulation to influence cell behavior associated with proliferation, differentiation, and migration have provided a better understanding of the underlying mechanisms of electrical stimulation-based clinical treatments and improved tissue-engineered products through electro-bioreactor technologies. Here, we present a novel device for delivering direct current (DC) electrical stimulation (ES) to cultivated cells in vitro. Our simplified electro-bioreactor is customized for applying DC electrical current simultaneously in six individual tissue culture wells. The design overcomes previous experimental replicate limitations, thus reducing experimental time and cost.

An ever-increasing number of diseases and tissue defects benefit from treatments that combine cell therapy and regenerative medicine technologies. In most applications, cells are initially isolated, expanded, or differentiated in 2-D or 3-D in vitro environments and then transplanted to the treatment site $(1-3)$. For the initial preparation, cells may be grown in standard 2-D culture dishes in $\mathrm{CO}_{2}$ incubators or in 3-D scaffolds in bioreactors that more closely mimic tissuespecific physiological environments.

Endogenous electrical fields were first described in damaged and healthy tissues more than a half century ago. Subsequently, investigators studied current pattern characteristics that suggested injury might change the electrophysiology of tissue repair (4-7). To study and understand the mechanisms of action of electrical fields in vivo, it is essential to mimic electrical activity in vitro. Recent in vivo and in vitro studies have shown that low voltage electrical stimulation (ES) plays an important role in regulating cell proliferation, differentiation, adhesion, matrix formation, and migration (8-11).

For in vitro experiments, a common method for delivering DC electrical stimulation to cells consists of a standard culture plate that delivers ES through salt bridges submerged in the culture media. The salt bridges separate cells from metallic electrodes to prevent the introduction of electro- chemical byproducts and $\mathrm{pH}$ changes to the media (12). While many valuable studies have been performed using this type of chamber, it is not easy to set up for the following reasons: the working area is small and limits the number of cells that can be studied in a single setting; the time that cells can be exposed to ES is limited because the concentration and heat differences between the bridge contents and the media drives the diffusion of salt and temperature into the media and vice-versa; it is difficult to run several chambers simultaneously; and it is challenging to maintain sterility. Furthermore, devices used to deliver DC electrical stimulation in the clinical setting, such as the BioMet system, use metallic electrodes rather than salt bridges, thus making results obtained during in vitro and in vivo experiments difficult to correlate (13).

In a few select studies, metallic or carbon electrodes have been used (14); however, these studies used single Petri dishes as chambers, limiting the working area and the number of cells and experiments that can be run in duplicate, thus hindering reproducibility. Here we present a device for exposing cells to electrical stimulation that was designed and developed to overcome these problems. The design consists of platinum electrodes secured to the top lid of a cell/tissue culture chamber that fits on a standard 6-well cell/ tissue-culture plate $(128 \times 85 \times 22 \mathrm{~mm}$; Internal $\varnothing 33.78 \mathrm{~mm}$; growth surface $8.960 \mathrm{~mm}$ ), permitting easy handling and sterilization and minimal medium evaporation (Figure 1A). Electrodes, made of $99.99 \%$ pure platinum wire (Junker Edelmetalle, Waldbüttelbrunn, Germany) and measuring 50 $\mathrm{mm}$ in length, $1 \mathrm{~mm}$ gauge with a single 21-29 mm long end, were bent into an L-shape. Two electrodes are placed precisely $25 \mathrm{~mm}$ apart inside each culture well of a standard 6-well cell culture plates (TPP, Trasadingen, Switzerland, firmly fixed to the lid, and hermetically sealed with silicone

\section{METHOD SUMMARY}

Here we present a novel in vitro tool for exposing progenitor/stem cells to electrical stimulation for inducing differentiation, migration, proliferation, electrotaxis, and polarization of cells. This device is applicable to various types of biological and biomedical studies that investigate the effects of bioelectrical stimulation and its applications. 
(A)

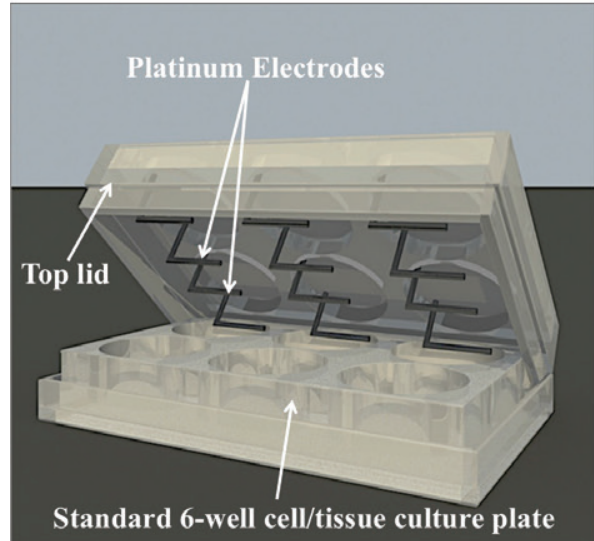

(B)

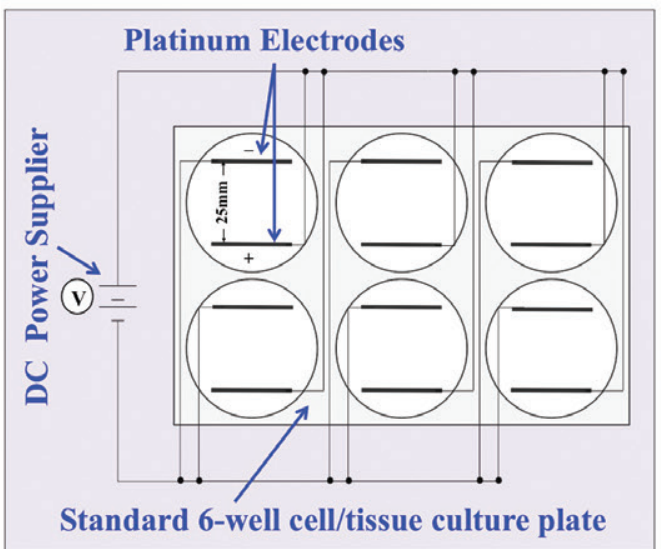

(C)

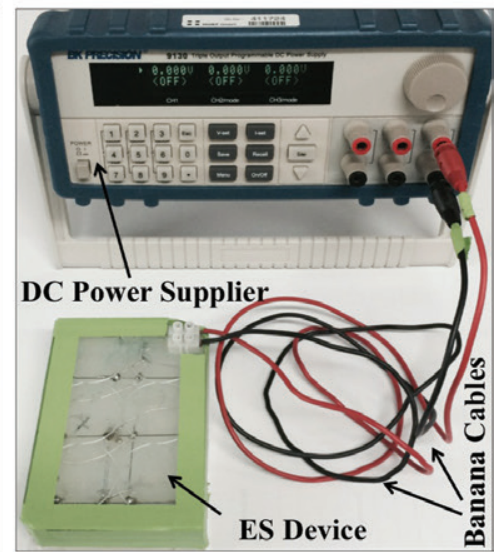

Figure 1. Electrical stimulation (ES) chamber. (A) ES device design of the chamber with L-shaped platinum electrodes incorporated into the lid of a standard 6-well plate. (B) Electric circuit diagram of the chamber. (C) ES device connected to the power supply with cables equipped with banana clips, facilitating transfer to an incubator while remaining connected.

glue. The tips of the electrodes, which protrude from the lid, are soldered to silver-coated copper wires in a parallel circuit (Figure 1B) and connected to a standard electric power supply (e.g., Triple Output Programmable DC Power Supplier (Supply-Model 9130; B\&K Precision, Yorba Linda, CA) by means of banana connectors. The amount of electricity and its pattern of application is tunable by adjusting the power supply, (Figure 1C). For sterilization, the chamber was flooded in $70 \%$ ethanol for $10 \mathrm{~min}$, washed in sterile calciumand magnesium-free phosphate buffer saline (DPBS; Gibco, Gaithersburg, MD), and then exposed to UV light in a cell culture laminar flow hood overnight.
This sterilization procedure rendered the containers free of eubacteria, as confirmed using a PCR kit for detection of eubacteria in cell culture (Bacteria Test Kit; PromoKine, Heidelberg, Germany).

To demonstrate the usefulness of our ES delivery device, we delivered ES to mesenchymal stem cells for a period of 7 days and assessed the effects on osteogenic differentiation. All tests were run at least in triplicate. Briefly, SpragueDawley (SD) rat adipose-derived mesenchymal stem cells (RASMD-01001; Cyagen, Santa Clara, CA) were thawed, cultured, and expand according to the provider's instructions. Cells were cultured at a density of $2.5 \times 10^{4} \mathrm{cell} / \mathrm{cm}^{2}$ until $80 \%$ confluent and then expanded over 4 passages. Passage 5 cells were seeded in 6-well cell culture plates (TPP) at a density of $10^{4} \mathrm{cell} / \mathrm{cm}^{2}$ at $37^{\circ} \mathrm{C}, 5 \%$ $\mathrm{CO}_{2}$, and $3 \%$ oxygen in a humidified incubator. The basal culture media was changed initially to osteogenic media containing $10^{-7} \mathrm{M}$ dexamethasone, 10 $\mathrm{mM} \beta$-glycerophosphate, and $0.05 \mathrm{mM}$ ascorbic acid-2-phosphate (SigmaAldrich, Hamburg, Germany) 1 day after seeding and then twice weekly. After 1 day of cultivation, the lids of the culture plates were exchanged with ES device lids (equipped with electrodes) and connected to the power supply. Applying 2.2 V DC, (as indicated on the power supply) resulted in the delivery
(A)

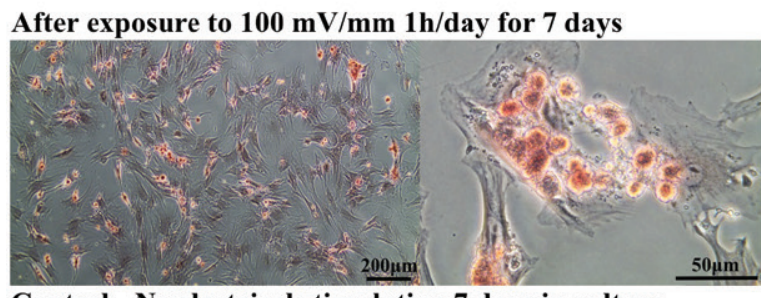

Control - No electrical stimulation 7 days in culture

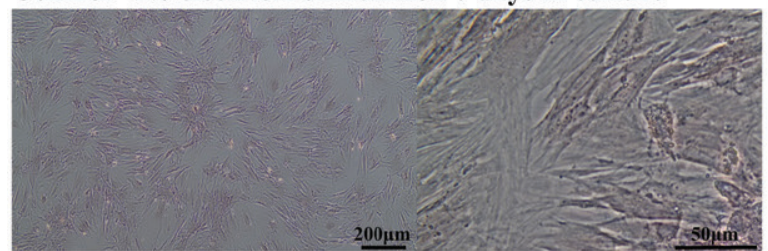

(B)

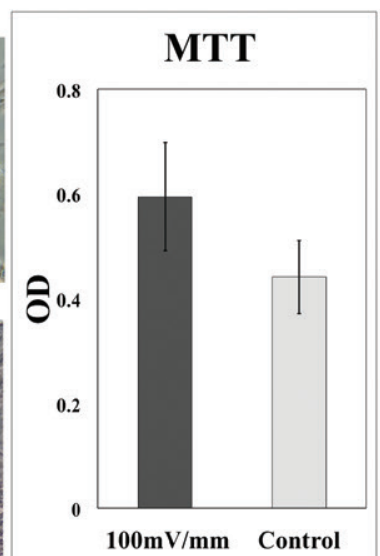

(C)

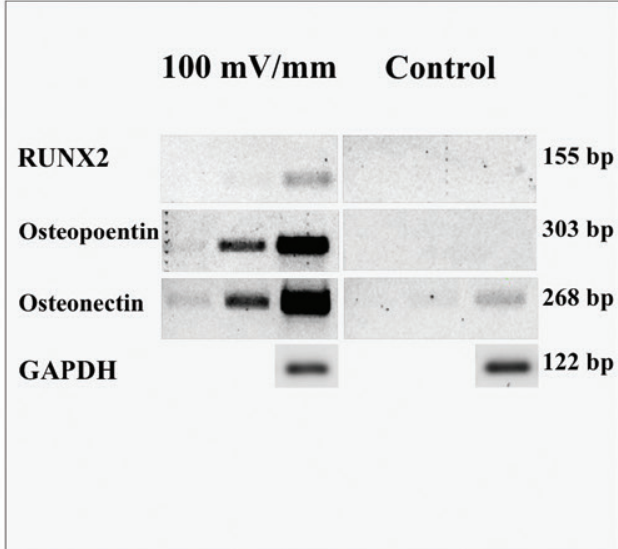

Figure 2. Electrical stimulation (ES) of rat adipose-derived mesenchymal stem cells (AT-MSCs) for 7 days. (A) Alizarin red staining of rat AT-MSCs exposed to $100 \mathrm{mV} / \mathrm{mm}$ for 7 days ( $1 \mathrm{~h} /$ day) versus controls (no electrical stimulation). (B) MTT cytotoxicity results confirm that the ES chamber has no toxic effect on cells. (C) Confirmation of osteogenic differentiation by semiquantitative RT-PCR. Runx2, osteopontin, and osteonectin mRNAS were expressed by the cells exposed to electrical stimulation, while there was no significant mRNA expression in the control group. 
of a $100 \mathrm{mV} / \mathrm{mm}$ DC field in each cell culture well. Cells were exposed to DC ES 1 h/day for 7 days and later compared with controls. At the end of each ES exposure time, the ES device lids equipped with electrodes were exchanged with the original lids. The ES device lids were cleaned and sterilized for use the next day.

At the end of the 7-day experiment, cells were washed twice with PBS, fixed with 4\% paraformaldehyde (SigmaAldrich) and stained with Alizarin Red $S$ (Sigma-Aldrich) for $30 \mathrm{~min}$. Figure 2A demonstrates positive staining for adipose-derived mesenchymal stem cells (AT-MSCs) exposed to $100 \mathrm{mV} /$ $\mathrm{mm}$ electrical stimulation. No calcium deposition was detected in control cells not exposed to ES.

To confirm that the metallic electrodes were not exposing cells in the culture media to possible cytotoxic effects due to oxidation-reduction and electrochemical reactions, cell viability and metabolic activity were assessed using the MTT assay (Kit I MTT; Roche Diagnostics, Mannheim, Germany) after 7 days exposure to ES (100 mV/ $\mathrm{mm}, 1 \mathrm{~h} /$ day). Samples from each well were transferred to a 96-well plate, and absorbance was read at $500 \mathrm{~nm}$ using a microplate reader (Infinite 200 Pro; TECAN, Männedorf, Switzerland). Results showed that there was no significant difference in viability between cells exposed to the metallic electrodes and electricity when compared with corresponding controls with no exposure (Figure 2B).

Osteogenic differentiation of ES-exposed cells was confirmed by evaluation of osteogenic marker expression using semiquantitative RT-PCR. Briefly, RT-PCR amplifications with primers specific to osteopontin, osteonectin, and RunX2 mRNAs were done using 100 ng cDNA. Total RNA (100 ng) was isolated from cells with Trizol (TRI-Reagent; Sigma-Aldrich), treated with DNase I (New England Biolabs Inc., Ipswich, MA), and reverse transcribed (iScript cDNA Synthesis Kit; Bio-Rad Laboratories Inc., Hercules, CA). PCR products were analyzed using gel electrophoresis and Image
Lab Software. GAPDH was used as a reference gene. Results show that RunX2 and osteopontin mRNAs were expressed only in electrically stimulated cells. Although detectable in both ES and controls, osteonectin mRNA transcripts were dramatically higher in the electrically stimulated cells. These data support alizarin red staining results and confirm the significant intensification in expression of osteogenic markers in AT-MSCs treated by DC ES (Figure 2C).

The stimulation paradigm (100 mV/ $\mathrm{mm}$ for $1 \mathrm{~h}$ ) was chosen as an effective regime to promote cell differentiation. Experiments for longer time periods (6, 8,24 , and $48 \mathrm{~h}$ ) revealed that cells were still intact throughout the culture plate, despite morphological changes near the electrode. Although longer exposure times $(>24 \mathrm{~h}$ ) with the same voltage increased the rate of evaporation of the medium due to non-dynamic medium flow in the system, this could be overcome by changing or adding medium. Based on these observations we believe that, continuous stimulation

\section{RNAzol ${ }^{\circledR}$ RT Column Kit}

Universal RNA isolation kit from Molecular Research Center

The market leader providing the best RNA isolation methods for over 25 years

Fast and dependable isolation

Highest yield and purity

No DNase treatment necessary
Process small or large samples

Up to $\mathbf{3 0 0}$ mg RNA per column

Variety of solid or liquid samples

\section{Specialized protocols for the isolation of:}

Total RNA containing all cellular RNA - Large RNA containing mRNA, rRNA and non-coding RNA Small RNA containing micro RNA

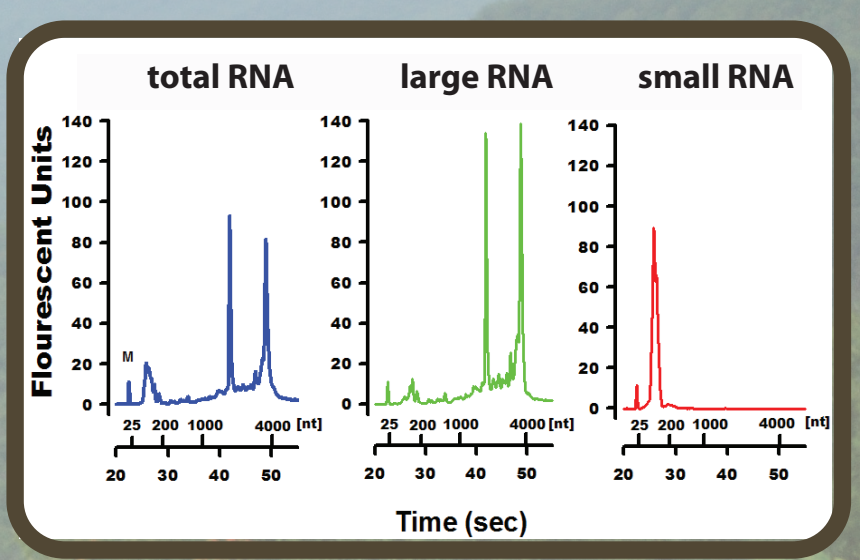


with $100 \mathrm{mV} / \mathrm{mm}$ would be possible. In addition to longer periods of exposure, we have also tested higher voltages, such as $200 \mathrm{mV} / \mathrm{mm}$, which caused the culture medium to split into two phases due to water electrolysis. Consequently, we believe that by using this device, cells can be exposed safely for longer time periods (when monitoring the medium to counter evaporation) and that the voltage should be kept below $200 \mathrm{mV} / \mathrm{mm}$.

An increasing number of in vitro studies in the literature focus on the effects of electrical stimulation on cells. The experiment presented here shows that ES delivered with this device causes a 3-fold increase in the rate of osteogenic differentiation of mesenchymal stem cells in comparison with non-stimulated cells. These results demonstrate the device's ability to generate meaningful data. In addition, the larger working area in this design not only provides a higher sample volume but also makes it possible to use 3-D cultures in future studies. We believe that this device could be a useful tool for studying the effects of ES in vitro on different types of cells. In conclusion, the purpose-built electrical stimulation device presented here enables researchers to expose cells to ES with an easy to use, re-usable, adjustable, and inexpensive chamber that can be applied to a variety of different experimental protocols.

\section{Author contributions}

S.M. designed and performed the experiments, analyzed data, and co-wrote the paper. L.L. designed the experiments, analyzed the data, and co-wrote the paper. J.B. administered the experiment and co-wrote the paper.

\section{Acknowledgments}

This study was supported by $\mathrm{AO}$ foundation, Switzerland Start-Up Grant S-14-03H and the Friedrichsheim Foundation (Stiftung Friedrichsheim) in Frankfurt/Main, Germany.

\section{Competing interests}

The authors declare no competing interests.

\section{References}

1. Caplan, A.I. 2005. Review: Mesenchymal stem cells: cell-based reconstructive therapy in orthopedics. Tissue Eng. 11:1198-1211.

2. Emmert, M.Y., R.W. Hitchcock, and S.P. Hoerstrup. 2014. Cell therapy, 3-D culture systems and tissue engineering for cardiac regeneration. Adv. Drug Deliv. Rev. 69-70:254-269.

3. Cancedda, R., B. Dozin, P. Giannoni, and R. Quarto. 2003. Tissue engineering and cell therapy of cartilage and bone. Matrix Biol. 22:81-91.

4. McCaig, C.D., B. Song, and A.M. Rajnicek. 2009. Electrical dimensions in cell science. J. Cell Sci. 122:4267-4276.

5. Gerard, R.W. 1942. Electrophysiology. Annu. Rev. Physiol. 4:329-358.

6. Borgens, R.B. 1984. Endogenous ionic currents traverse intact and damaged bone. Science 225:478-482.

7. Nuccitelli, R. 1992. Endogenous ionic currents and DC electric fields in multicellular animal tissues. Bioelectromagnetics Suppl 1:147-157.

8. Griffin, M. and A. Bayat. 2011. Electrical stimulation in bone healing: critical analysis by evaluating levels of evidence. Eplasty 11:e34.

9. Banks, T.A., P.S.B. Luckman, J.E. Frith, and J.J. Cooper-White. 2015. Effects of electric fields on human mesenchymal stem cell behavior and morphology using a novel multichannel device. Integr. Biol. 7:693-712.

10. McCaig, C.D., A.M. Rajnicek, B. Song, and M. Zhao. 2005. Controlling cell behavior electrically: current views and future potential. Physiol. Rev. 85:943-978.

11. McCaig, C.D. and M. Zhao. 1997. Physiological electrical fields modify cell behavior. Bioessays 19:819-826.

12. Song, B., Y. Gu, J. Pu, B. Reid, Z. Zhao, and M. Zhao. 2007. Application of direct current electric fields to cells and tissues in vitro and modulation of wound electric field in vivo. Nat. Protoc. 2:1479-1489.

13. Simon, B.J. 2012. U.S. Patent No. 8,145,319. Washington, DC: U.S. Patent and Trademark Office.

14. Hronik-Tupaj, M., W.L. Rice, M. Cronin-Golomb, D.L. Kaplan, and I. Georgakoudi. 2011. Osteoblastic differentiation and stress response of human mesenchymal stem cells exposed to alternating current electric fields. Biomed. Eng. Online 10:9.

Received 17 July 2015; accepted 28 September 2015.

Address correspondence to Sahba Mobini (present address): School of Materials, Faculty of Engineering and Physical Sciences, University of Manchester, Manchester M1 3AL, UK. E-mail: sahba.mobini@manchester.ac.uk

To purchase reprints of this article, contact: biotechniques@fosterprinting.com 